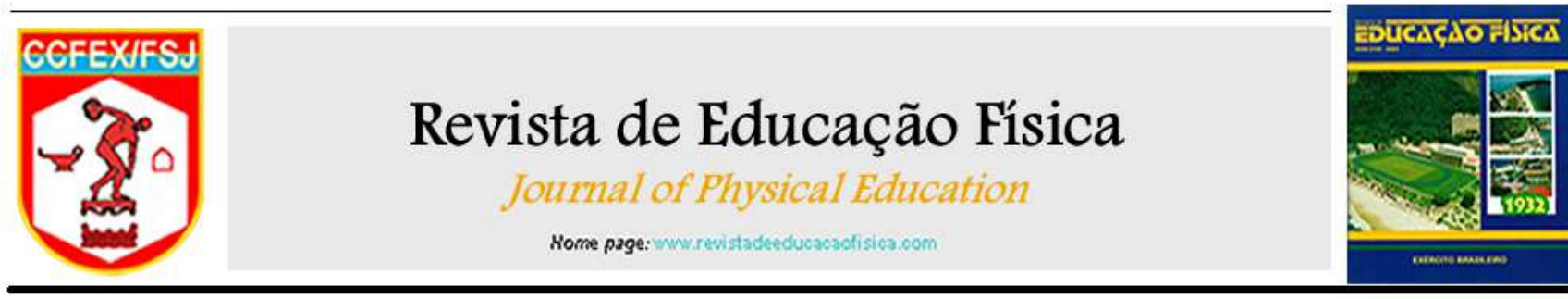

Artigo Original

Original Article

\title{
Tomada de decisão, aptidão física e idade no desempenho de equipes de corrida de aventura
}

\section{Decision making, physical fitness and age in the performance of adventure racing teams}

\author{
Fernanda Dapper Coelho ${ }^{1}$ Esp, Márcio de Avila Palermo ${ }^{1}$ Esp, Antônio Fernando Araújo Duarte ${ }^{2}$ PhD, \\ Marcelo Coertjens ${ }^{3 \S} \mathrm{MS}$, Luiz Fernando Martins Kruel ${ }^{1} \mathrm{PhD}$
}

Recebido em: 26 de agosto de 2015. Aceito em: 14 de fevereiro de 2016.

Publicado online em: 30 de março de 2016.

\section{Resumo}

Introdução: O desempenho de equipes de corrida de aventura (CA) parece sofrer influência não apenas de variáveis de aptidão física, mas também da experiência e da capacidade de tomada de decisão de seus navegadores.

Objetivo: 0 objetivo deste estudo foi avaliar a associação de nível de aptidão física (variáveis fisiológicas cardiopulmonares) e idade com desempenho em tomada de decisão (performance) e desempenho competitivo em equipes de CA.

Métodos: Oito navegadores foram submetidos a um teste de Tomada de Decisão (TD) cognitivo, antes (TD1) e após (TD2) a realização de um teste de esforço progressivo máximo em esteira. Para comparação das médias dos resultados do TD foi utilizado Teste $\mathrm{t}$ de Student para amostras pareadas e Teste de Wilcoxon. Para as associações, utilizaram-se correlações de Pearson e Spearman $(\mathrm{p}<0,05)$.

Resultados: As variáveis fisiológicas e o desempenho na competição apresentaram baixa correlação $(p>0,05)$. Idade apresentou bons índices de correlação com os resultados do TD2

Pontos-Chave Destaque

- Navegadores mais velhos apresentaram menor quantidade de erros em tomada de decisão.

- Equipes compostas por atletas mais velhos apresentaram melhor desempenho.

- Erros influenciam mais o desempenho do que acertos em corrida de aventura. (PONTOS: $r=-0,84$; ACERTOS: $r=-0,86$; ERROS: $r=-0,77$; $p<0,01$ ) e com o desempenho das equipes $(r=-0,71$, $\mathrm{p}<0,05$ ).

Conclusão: A idade dos navegadores pode ser um fator que influencia o desempenho final das equipes de CA, pois, é provável que os atletas com mais idade possuam mais experiência de competição, que somada a fatores psicológicos, podem influenciar a tomada de decisão de modo a escolher melhores percursos durante as competições, contribuindo para o desempenho competitivo da equipe.

Palavras-chave: aptidão física, psicofisiologia, corrida, orientação.

Abstract
Introduction: The performance of adventure racing (AR) seems to be influenced not only by physical fitness variables, but also by the experience and decision-making capacity of the navigators.

\footnotetext{
${ }^{\S}$ Autor correspondente: Marcelo Coertjens - email: coertjens@hotmail.com

Afiliações: ${ }^{1}$ Universidade Federal do Rio Grande do Sul. Escola de Educação Física. Grupo de Pesquisa em Atividades Aquáticas e Terrestres. Porto Alegre, RS. ${ }^{2}$ Instituto de Pesquisa de Capacitação Física do Exército - Rio de Janeiro, RJ. ${ }^{3}$ Universidade Federal do Piauí. Campus Ministro Reis Velloso. Departamento de Ciências da Saúde/Fisioterapia. Parnaíba, PI.
} 
Objective: The objective of this study was to evaluate the association of physical fitness level (cardiopulmonary physiologic variables) and age with performance in decision-making and competitive performance in AR teams.

Methods: Eight navigators underwent to a Decision Making (DM) test, before (DM1) and after (DM2) a maximal graded exercise test. To compare the average of the results of DM was used the Student $t$ test for paired samples and Wilcoxon test. For associations were used Spearman and Pearson correlations $(\mathrm{p}<0.05)$.

Results: Physiological and performance variables in the competition presented low correlation ( $p>0.05$ ), navigators age showed good correlation coefficients with DM2 (POINTS: $r=-0.84$; SETTING: $r=-0.86$; ERROR: $r=-0.77, p<0.01$ ) and team performance $(r=-0.71, \mathrm{p}<0.05)$.

Conclusion: Navigators age may be a factor that influences the performance of AR because it is probable that older competitors presents larger technical experience, which coupled with psychological factors appear to influence the decision-making leading to choose best routes during competitions contributing to the team's performance.

Keywords: physical fitness, psychophysiology, running, orientation sport.

\section{Tomada de decisão, aptidão física e idade no desempenho de equipes de corrida de aventura}

\section{Introdução}

O exercício físico é considerado um dos muitos exemplos de estímulo que desencadeiam estresse físico e psicológico sobre o organismo humano. Isso se deve porque ele induz consideráveis mudanças fisiológicas no sistema imune e neuroendócrino semelhante à resposta comum do estresse $(1,2)$.

Diversos fatores podem estar associados à resposta imune e neuroendócrina ao estresse durante o exercício físico. Dentre eles, estão a idade e o sexo do indivíduo(3), o grau de treinamento físico(2), o estado nutricional(4) e a temperatura e umidade local durante $o$ exercício físico(5). O perfil psicológico e o estado de humor do atleta têm sido sugeridos, também, como fatores capazes de induzir o estresse e determinarem o desempenho de atletas, sobretudo em esportes nos quais, em conjunto com as exigências físicas, haja maior demanda de aspectos cognitivos e psicológicos(4,5-8).

Um exemplo de esporte que submete seus praticantes a diferentes fatores responsáveis pela resposta ao estresse é a corrida de aventura $(\mathrm{CA})(1,9,10)$. Este esporte geralmente formado por equipes compostas de quatro competidores é realizado em ambiente natural de forma a submeter os atletas a condições climáticas e de terreno desfavoráveis, como chuva, umidade, frio, vento e grandes desníveis de terreno. Compreende diferentes modalidades esportivas sendo as principais o trekking (caminhada em trilhas), as técnicas verticais de escalada, a corrida de orientação e o mountain bike, que são realizadas aleatoriamente em um percurso que varia entre 40 e $500 \mathrm{~km}$. Em cada quarteto há um navegador que tem como função orientar o percurso de sua equipe durante a prova através de carta topográfica e bússola. A equipe deve percorrer o trajeto escolhido pelo navegador no menor tempo possível, passando por todos os postos de controle (PCs) estipulados pelos organizadores da prova(11).

Além do desgaste físico sofrido pela equipe para percorrer $\mathrm{o}$ trajeto $\mathrm{e}$ superar os obstáculos naturais, a responsabilidade pela tomada de decisão na orientação é um fator a mais que influencia a resposta ao estresse e o desempenho físico do indivíduo responsável pela navegação $(7,8,12)$. A capacidade de tomada de decisão a respeito do caminho a seguir torna-se relevante, pois o navegador 
deve escolher o melhor percurso para passar por todos os PCs o mais rápido possível, com o mínimo de erros e desgaste físico(12).

Diversos estudos têm constatado a interação da aptidão física com as respostas psicofisiológicas, demonstrando que os indivíduos que apresentam melhor condicionamento aeróbio obtêm menores respostas fisiológicas ao estresse(4-6). A relação dos aspectos psicológicos $\mathrm{e}$ emocionais com a aptidão física mostra-se, portanto, determinante para o desempenho de toda a equipe, visto que se o navegador não estiver com um bom nível de aptidão física, o estresse poderá afetar negativamente a sua tomada de decisão, prejudicando a equipe.

Palermo et al.(13), encontraram baixa correlação entre a aptidão física de navegadores e dos demais membros da equipe de CA com o desempenho em competições (entre -0,34 e -0,18). Esta constatação foi obtida a partir de variáveis consideradas preditoras de desempenho tais como 0 consumo máximo de oxigênio $\left(\mathrm{VO}_{2 \text { máx }}\right)$ e variáveis obtidas no segundo limiar ventilatório $\left(2^{\circ} \mathrm{LV}\right): \mathrm{o}_{2} \mathrm{VO}_{2}$ correspondente ao segundo limiar ventilatório $\left(\mathrm{VO}_{2} 2^{\circ} \mathrm{LV}\right), \mathrm{o}$ percentual (\%) que este se encontra em relação ao $\mathrm{VO}_{2 \text { máx }}\left(\%{ }^{\circ} \mathrm{LV}\right)$ e o tempo de teste correspondente ao segundo limar ventilatório (Temp_2 $\left.{ }^{\circ} \mathrm{LV}\right)(14-17)$. O coeficiente de correlação encontrado entre a ordem de classificação dessas equipes em uma competição de CA e a idade dos navegadores foi de $-0,77$. Entretanto, o estudo não contemplou aspectos psicofisiológicos relacionados ao desempenho. A lacuna permanece.

Dessa forma, é plausível que dentre os navegadores com níveis de aptidão física semelhantes, aqueles mais velhos sejam, provavelmente, mais experientes e, portanto, teriam mais sucesso na prática da navegação. Tal sucesso poderia ser atribuído a menores efeitos de estresse físico e mental ou por serem mais bem sucedidos na tomada de decisão. Isso favoreceria um melhor desempenho para sua equipe durante as provas. O objetivo deste estudo foi avaliar a associação de nível de aptidão física (variáveis fisiológicas cardiopulmonares) e idade com desempenho em tomada de decisão (performance) e desempenho competitivo em equipes de $\mathrm{CA}$.

\section{Métodos}

O presente trabalho foi realizado após um período de competições de corrida de aventura é do tipo transversal correlacional e retrospectivo. Neste estudo, os resultados de um teste psicofisiológico realizado em navegadores de equipes de $\mathrm{CA}$ foram coletados antes e após um teste de esforço progressivo máximo e correlacionados com seus parâmetros fisiológicos, com idade e desempenho de suas equipes em competição.

\section{Amostra}

A amostra deste estudo foi composta por oito indivíduos do sexo masculino, navegadores das equipes que participaram de uma etapa de um circuito competitivo de CA, com distância média de $60 \mathrm{~km}$, modalidade quarteto misto, composto por 3 homens e 1 mulher. Para cada equipe havia apenas um navegador. As modalidades que constituíram as provas foram orientação, mountain bike, corrida, canoagem e técnicas verticais de escalada. Todos os participantes eram voluntários, foram esclarecidos previamente a respeito dos objetivos e procedimentos deste estudo e assinaram Termo de Consentimento Livre e Esclarecido aprovado pelo Comitê de Ética local, conforme a resolução 196/96 do Conselho Nacional de Saúde.

Todos os indivíduos foram orientados a não ingerir substâncias que contivesse cafeína ou qualquer outra bebida que contivesse estimulantes nas últimas $12 \mathrm{~h}$ antes do teste. Foi orientado, também, que os indivíduos não realizassem nenhum tipo de exercício físico $24 \mathrm{~h}$ antes do teste e que procurassem ter uma noite de sono tranquila. Os testes foram realizados entre 15 a 20 dias após a realização da competição.

\section{Procedimentos}

Os indivíduos foram submetidos, primeiramente, a um teste de diagnóstico psicofisiológico sob condições estressantes durante $5 \mathrm{~min}$ (TD1). Este teste foi realizado após os atletas permanecerem em repouso por $15 \mathrm{~min}$ (10 min na posição supina e $5 \mathrm{~min}$ sentados). Após este teste, os atletas permaneceram em repouso sentados durante 3 
min e, então, realizaram um teste de esforço progressivo máximo em esteira. Finalizado o teste de esforço físico, os indivíduos permaneceram por mais $3 \mathrm{~min}$ em repouso sentado e, em seguida, realizaram o TD2.

\section{Protocolo de testes}

\section{Teste psicofisiológico em tomada de} $\underline{\text { decisão }}$

Durante o teste psicofisiológico de tomada de decisão (TD) (Biofeedback 2000, Alemanha) o indivíduo é desafiado a relacionar as cores e as formas geométricas de figuras que aparecem no centro da tela do computador com uma lista de possibilidades localizada nas laterais da tela através de clicas no mouse. A velocidade com que essas figuras aparecem aumenta à medida que os indivíduos acertam. $O$ programa calcula continuamente a dificuldade do teste, adaptando-o de modo a permitir que o indivíduo alcance uma performance máxima final de cerca de 50\%. Dessa forma o indivíduo é submetido a um nível de estresse constante permitindo avaliar suas adaptações e reações psicofisiológicas, bem como seus erros e acertos sob situações estressantes. Ao final do teste, o programa informa os resultados do teste através de um relatório que consiste em: pontos atingidos (PONTOS), nível de dificuldade atingida (NÍVEL), total de acertos (ACERTOS), total de erros (ERROS) e performance (PERF): desempenho em tomada de decisão. Calculase PERF a partir da divisão dos pontos atingidos pelos pontos a serem atingidos, multiplicando esse resultado por 100. O cálculo dos pontos atingidos é baseado no total de acertos do teste e no nível de dificuldade onde foram feitos esses acertos. Ou seja, cada acerto vale diferentes pontos que são determinados pelo nível de exigência em que o indivíduo está sendo submetido. Sendo assim, quanto mais alto o nível de dificuldade atingido, maior é o ponto de um acerto. Para minimizar o efeito da aprendizagem, todos os indivíduos realizaram previamente uma familiarização com o teste de tomada de decisão.

\section{$\underline{\text { Teste físico }}$}

O teste de esforço físico na esteira consistiu de um teste progressivo máximo utilizando um protocolo de rampa com aumentos da velocidade e da inclinação da esteira (IMBRAMED 10200ATL, Porto Alegre). O teste iniciava com velocidade a $6 \mathrm{~km} * \mathrm{~h}-1$ e nível de inclinação da esteira de $1 \%$. Foram realizados aumentos progressivos na velocidade da esteira de $0,5 \mathrm{~km} * \mathrm{~h}-1$ a cada 30 s e na inclinação de $1 \%$ a cada 30 s a partir da velocidade de $12 \mathrm{~km} * \mathrm{~h}-1$. A velocidade ficava constante a partir de $17 \mathrm{~km} * \mathrm{~h}-1$. O término do teste era determinado por decisão voluntária do executante ou quando eram observados sintomas de limite máximo de esforço e incapacidade de manter a potência de trabalho. Os resultados deste teste foram considerados satisfatórios quando os indivíduos atingiam os seguintes critérios: valores de frequência cardíaca máxima maior ou igual a $90 \%$ da frequência cardíaca máxima prevista pela idade e taxa de troca respiratória maior do que 1.1(18).

O teste de esforço físico teve como objetivo determinar o $\mathrm{VO}_{2} 2^{\circ} \mathrm{LV}, \quad \mathrm{o} \quad \% 2^{\circ} \mathrm{LV}, \quad \mathrm{o}$ Temp_ $2^{\circ} \mathrm{LV}$ e o $\mathrm{VO}_{2 \text { máx }}$ dos navegadores. $\mathrm{O}$ $\mathrm{VO}_{2 \text { máx }}$ foi determinado como sendo o valor de consumo de oxigênio $\left(\mathrm{VO}_{2}\right)$ relativo mais alto encontrado entre as médias de intervalos de $30 \mathrm{~s}$ coletado continuamente utilizando circuito de espirometria aberta (Medical Graphics CPX/D, Arizona, EUA). A análise do $\mathrm{VO}_{2} 2^{\circ} \mathrm{LV}$ foi realizada por dois avaliadores experientes a partir do processamento dos dados "breath by breath" (respiração a respiração) pela média de cinco dados a cada sete, excluindo-se os valores mais alto e o mais baixo para $\mathrm{VO}_{2}$, produção de gás carbônico $\left(\mathrm{VCO}_{2}\right)$, ventilação minuto (VE) e pressão expiratória de gás carbônico $\left(\mathrm{PETCO}_{2}\right)$. Para sua determinação, tomava-se como referência o ponto no qual se verificava um aumento exponencial da curva de ventilação minuto (VE), quando ocorresse um aumento do $\mathrm{VE} / \mathrm{VCO}_{2}$ ou quando ocorresse uma queda na $\mathrm{PETCO}_{2}$ (19). Estudos têm verificado que a utilização de métodos combinados para determinação dos limiares ventilatórios resulta em maior acurácia(20). Antes de cada teste foram realizados os procedimentos básicos de calibração manual 
do sistema de espirometria através de um êmbolo de 3 litros e do sistema de analise de gases por meio de uma mistura de gases de conteúdo conhecido de acordo com as normas do fabricante.

\section{Análise estatística}

Os dados foram apresentados através da estatística descritiva (média e desvio-padrão). A normalidade e dos dados foi avaliada pelo teste de Shapiro-Wilk $(\mathrm{p}>0,05)$. Foi utilizado Teste $t$ de Student para amostras dependentes para comparar os resultados obtidos no TD realizado antes e depois do teste de esforço físico. As variáveis que não apresentaram normalidade foram comparadas através do Teste de Wilcoxon. Para verificar a correlação entre as variáveis analisadas foi utilizada a correlação linear produto momento de Pearson, para os dados que apresentaram normalidade. Para analisar as variáveis ordinais (desempenho das equipes na competição) e aquelas que não apresentaram normalidade, utilizou-se a correlação linear de Spearman. O nível de significância adotado foi $\mathrm{p}<0,05$. O pacote estatístico utilizado foi o SPSS v.s. 14.0 for Windows.

\section{Resultados:}

Os atletas avaliados apresentaram estatura média de 178,2 $\pm 9,2 \mathrm{~cm}$ e uma massa corporal média de 74,4 $\pm 5,9 \mathrm{~kg}$. A média de idade foi de 27,6 \pm 4,8 anos. Na Tabela 1, pode-se observar os resultados da estatística descritiva de idade e de aptidão física. A duração média do teste progressivo máximo foi de 09:57 $\pm 1: 27 \mathrm{~min}$.

Na Tabela 2, observa-se que as médias de pontos, acertos e erros foram maiores em TD2 que TD1 $(p<0,05)$. Todas as variáveis verificadas apresentaram normalidade em sua distribuição $(p>0,05)$, com exceção das variáveis ACERTOS_1 e PERF_2. Observouse um discreto aumento no desempenho (performance) em tomada de decisão em TD2. Todavia, não houve significância estatística $(\mathrm{P}=0,75)$.

Os resultados apresentaram fracos coeficientes de correlação $(p>0,05)$ entre $o$ desempenho das equipes de $\mathrm{CA}$ em competição com o $\mathrm{VO}_{2 \text { máx }}(\mathrm{r}=-0,32)$ e as variáveis fisiológicas avaliadas durante $\mathrm{o}$ $2^{\circ} \mathrm{LV}$ dos navegadores $\left(\mathrm{VO}_{2} 2^{\circ} \mathrm{LV}: \mathrm{r}=0,25\right.$; $\% \_2^{\circ} \mathrm{LV}: \mathrm{r}=0,45$ e Temp_ $\left.2^{\circ} \mathrm{LV}: \mathrm{r}=0,44\right)$. Resultados semelhantes foram encontrados entre as variáveis fisiológicas e TD (TABELA 3).

Tabela 1 - Estatísticas descritivas de idade e variáveis fisiológicas dos navegadores e desempenho competitivo das respectivas equipes

\begin{tabular}{|c|c|c|c|c|c|c|}
\hline ID & Idade & $\begin{array}{l}\mathrm{VO}_{2} \\
\text { máx }\end{array}$ & $\begin{array}{c}\mathrm{VO}_{2} \mathbf{2}^{2} \\
{ }^{\mathbf{o}} \mathbf{L}\end{array}$ & $\begin{array}{c}\% 2^{\circ} \\
\mathbf{L V}\end{array}$ & $\begin{array}{l}\text { Temp } \\
2^{\circ} \mathrm{LV}\end{array}$ & DES \\
\hline $\bar{~} \overline{1}$ & 30 & 447,5 & 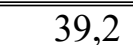 & 82,5 & $7: 10$ & $\overline{1^{\circ}}$ \\
\hline 2 & 28 & 46,3 & 38,1 & 82,3 & $8: 30$ & $3^{\circ}$ \\
\hline 3 & 31 & 52,7 & 50,0 & 94,9 & $9: 22$ & $4^{\circ}$ \\
\hline 4 & 36 & 46,7 & 42,6 & 91,2 & $7: 10$ & $5^{\circ}$ \\
\hline 5 & 23 & 51,0 & 48,3 & 94,7 & $9: 05$ & $7^{\circ}$ \\
\hline 6 & 27 & 51,0 & 44,9 & 88,1 & $9: 35$ & $8^{\circ}$ \\
\hline 7 & 25 & 44,8 & 42,6 & 95,1 & $9: 40$ & $12^{\circ}$ \\
\hline 8 & 21 & 46,3 & 42,1 & 90,9 & $7: 51$ & $16^{\circ}$ \\
\hline MD & 27,6 & 48,3 & 43,5 & 90 & $8: 32$ & \\
\hline$\pm \mathrm{dp}$ & $\pm 4,8$ & $\pm 2,9$ & $\pm 4,1$ & $\pm 5,3$ & $\pm 1: 02$ & \\
\hline
\end{tabular}

VO $_{2 \text { máx }}:$ consumo máximo de oxigênio (ml*kg*min-1); $\mathbf{V O}_{2 \_} 2^{\circ} \mathbf{L V}$ : consumo de oxigênio correspondente ao segundo limiar ventilatório $\left(\mathrm{ml} * \mathrm{~kg}^{*} \mathrm{~min}-1\right) ; \% \mathbf{\%} \mathbf{2}^{\mathbf{O}} \mathbf{L V}$ : percentual do $\mathrm{VO}_{2} 2^{\circ} \mathrm{LV}$ em relação ao $\mathrm{VO}_{2 \text { máx }}(\%)$; $\mathbf{T}$ emp $2^{\circ} \mathbf{L V}$ : tempo de teste correspondente ao segundo limiar ventilatório (min:seg); DES: Desempenho das equipes na competição.

Tabela 2 - Estatísticas descritivas das variáveis componentes do teste de Tomada de Decisão (TD) - antes (TD1) e depois (TD2) do teste de esforço físico dos navegadores

\begin{tabular}{lrrr}
\hline \multicolumn{1}{c}{ Variáveis } & \multicolumn{1}{c}{ TD1 } & \multicolumn{1}{c}{ TD2 } & P \\
\hline $\begin{array}{l}\text { PONTOS } \\
\text { (unid) }\end{array}$ & $18974,4 \pm$ & $22085,3 \pm$ & $\mathbf{0 , 0 3}$ \\
$\begin{array}{l}\text { NÍVEL } \\
\text { (unid) }\end{array}$ & $189,4 \pm 1,0$ & 4699,4 & \\
$\begin{array}{l}\text { ACERTOS } \\
\text { (unid) }\end{array}$ & $55,0 \pm 9,9$ & $62,0 \pm 12,2$ & $\mathbf{0 , 0 3}$ \\
$\begin{array}{l}\text { ERROS } \\
\text { (unid) }\end{array}$ & $103,2 \pm$ & $125,3 \pm 27,8$ & $\mathbf{0 , 0 1}$ \\
PERF (\%) & 14,2 & & 0,38 \\
\hline
\end{tabular}

PONTOS: pontos atingidos; NÍVEL: nível de dificuldade; ACERTOS: total de acertos; ERROS: total de erros; PERF: performance (desempenho no teste de Tomada de Decisão (TD); P: valores de $\mathrm{P}$ obtidos a partir do teste Teste $t$ de Student para amostras dependentes e Teste de Wilcoxon para as variáveis ACERTOS e PERF.

Bons coeficientes foram encontrados em relação à idade dos navegadores com os parâmetros obtidos no TD2 e com o desempenho das equipes em competição $(\mathrm{p}<$ 0,05) (FIGURA 1). 
Tabela 3 - Correlação de idade e variáveis fisiológicas com tomada de decisão (TD) antes e depois do teste físico e com desempenho competitivo em corrida de aventura (CA)

\begin{tabular}{|c|c|c|c|c|c|c|c|}
\hline Variáveis & Idade & $\mathrm{VO}_{2} 2^{\circ} \mathrm{LV}$ & $\mathrm{VO}_{2 \text { máx }}$ & $\begin{array}{c}\text { ACERTOS } \\
\text { TD1 }\end{array}$ & $\begin{array}{c}\text { ERROS } \\
\text { TD1 } \\
\end{array}$ & $\begin{array}{c}\text { ACERTOS } \\
\text { TD2 }\end{array}$ & $\begin{array}{c}\text { ERROS } \\
\text { TD2 } \\
\end{array}$ \\
\hline Idade & & & & & & & \\
\hline $\mathrm{VO}_{2} 2^{\circ} \mathrm{LV}$ & $-0,06$ & & & & 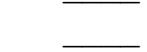 & & \\
\hline $\mathrm{VO}_{2 \text { máx }}$ & 0,07 & $0,81 *$ & & & & & \\
\hline ACERTOS_TD1 & $-0,50$ & $-0,32$ & 0,05 & & & & \\
\hline ERROS_TD̄1 & $-0,66$ & $-0,25$ & $-0,07$ & $0,89 * *$ & & & \\
\hline ACERTŌ_TD2 & - & 0,20 & 0,16 & $0,80^{*}$ & $0,82^{*}$ & & \\
\hline & $0,86^{* *}$ & & & & & & \\
\hline ERROS_TD2 & $-0,77^{*}$ & 0,38 & 0,17 & 0,45 & 0,61 & $0,92 * *$ & \\
\hline DES & $-0,71 *$ & 0,25 & $-0,32$ & $-0,07$ & 0,05 & 0,15 & 0,52 \\
\hline
\end{tabular}

$\mathbf{V O}_{2 \_} \mathbf{2}^{\circ} \mathbf{L V}$ : consumo de oxigênio correspondente ao segundo limiar ventilatório (ml*kg*min-1); $\mathbf{V O}_{2 \text { máx }}$ : consumo máximo de oxigênio (ml*kg*min-1); ACERTOS: total de acertos; ERROS: total de erros; DES: desempenho das equipes na competição. Significância estatística obtida pelo teste de correlação linear produto momento de Pearson e correlação linear de Spearman: * $\mathrm{p}<0,05 . * * \mathrm{p}<0,01$
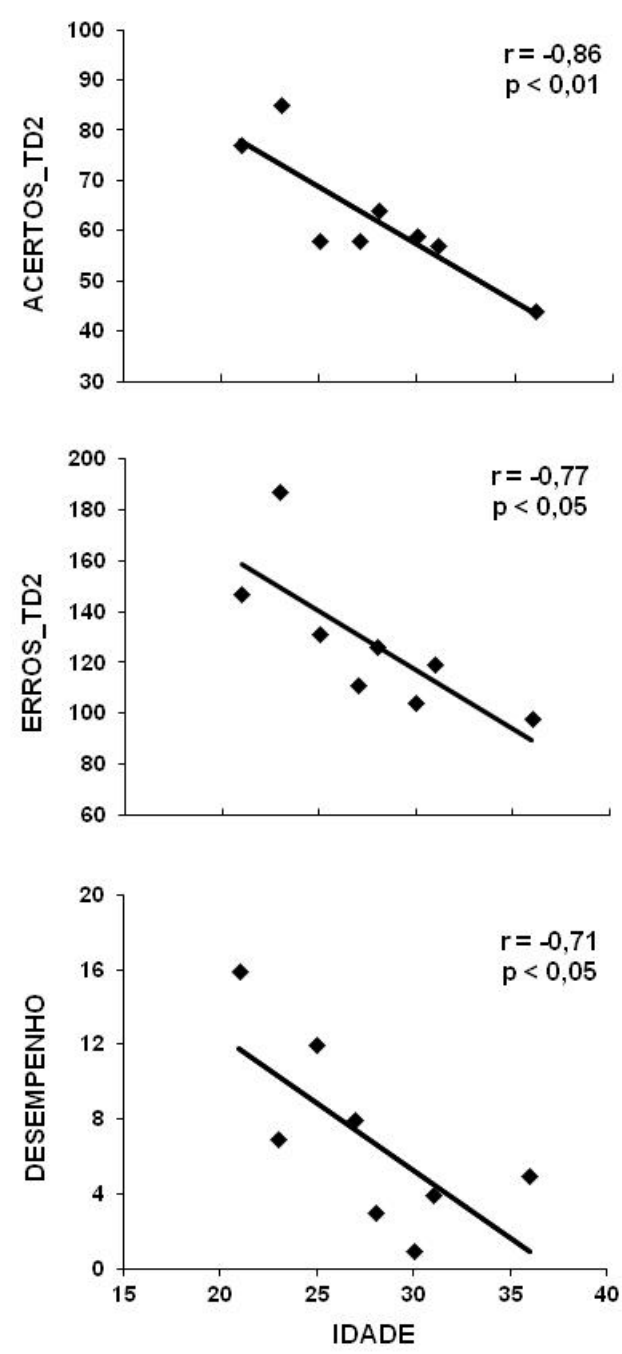

FIGURA 1: Correlação entre idade e variáveis fisiológicas obtidas durante teste de tomada de decisão (td) realizado após teste de esforço físico $(\operatorname{td} 2)$ e desempenho das equipes em competição

\section{Discussão}

O presente estudo teve como objetivo avaliar a associação de nível de aptidão física (variáveis fisiológicas cardiopulmonares) e idade com desempenho em tomada de decisão (performance) e desempenho competitivo em equipes de CA. Os achados do estudo indicam que, apesar da importância de uma preparação física adequada para as exigências das provas de CA, o desempenho final das equipes em uma competição pode sofrer significativa influência do desempenho (performance) em tomada de decisão do navegador.

De forma geral, o $\mathrm{VO}_{2 \text { máx }}$ e o $2^{\circ} \mathrm{LV}$ têm sido apontados como importantes marcadores fisiológicos de desempenho em esportes de longa distância(21-23).

Em estudos anteriores, foram encontradas boas correlações entre $\mathrm{VO}_{2 \text { máx }}$ e desempenho em corredores universitários $(\mathrm{r}=-0,76)(14)$, atletas de triatlo masculinos $(\mathrm{r}=-0,84)(24)$ e femininos $(r=-0,88)(17)$.

Em relação ao $2^{\circ} \mathrm{LV}$, a maior parte dos estudos tem encontrado boas correlações com desempenho competitivo ao analisarem o $\mathrm{VO}_{2} 2^{\circ} \mathrm{LV}$ de triatletas $(\mathrm{r}=-0,88)(14)$, o $\% 2^{\circ} \mathrm{LV}$ de maratonistas $(\mathrm{r}=-0,85)(15)$ e a Vel_2 ${ }^{\circ} \mathrm{LV}$ tanto de corredores $(\mathrm{r}=-0,96)(16)$ e $(\mathrm{r}=-0,89)(17)$ como de triatletas $(\mathrm{r}=$ $0,81)(14)$. Isto se deve, principalmente, por permitir ao atleta retardar a intervenção do metabolismo anaeróbio durante o aumento da intensidade de trabalho(15). Esta característica se mostra relevante, quando os atletas competem em provas em que a 
mudança de intensidade é constante, como é o caso de corredores de cross-country(25). No caso de CA, a importância de um alto $2 \mathrm{oLV}$ se fará presente, também, em momentos onde os terrenos com grandes desníveis e obstáculos naturais exigirão dos atletas executar curtas explosões de grande intensidade que necessitem de um rápido acesso a um grande volume de energia ou, então, evitar a diminuição do ritmo dos atletas durante a prova(11). Esta afirmação corrobora, para a importância de um alto 2oLV no desempenho final das equipes nestas competições. Nossos resultados, entretanto, apresentaram fracos coeficientes de correlação entre $\mathrm{o} \quad \mathrm{VO}_{2 \text { máx }}$ e as variáveis avaliadas durante o $2^{\circ} \mathrm{LV}$ dos navegadores com o desempenho de suas equipes. Resultados semelhantes foram encontrados nos estudos de Palermo et al.(13) que avaliaram as seis melhores equipes classificadas em um circuito composto por 5 etapas diferentes de CA. No estudo, 24 atletas de CA foram submetidos a um teste progressivo até a intensidade máxima em esteira. Os valores encontrados apresentaram fraca correlação da aptidão física dos atletas avaliados $\left(\mathrm{VO}_{2 \text { máx }}: \mathrm{r}=-0,34 ; \mathrm{VO}_{2} 2^{\circ} \mathrm{LV}: \mathrm{r}=\right.$ 0,$22 ; \% \_2^{\circ} \mathrm{LV}: \mathrm{r}=-0,14$; Temp_2 ${ }^{\circ} \mathrm{LV}: \mathrm{r}=$ $0,18)$ e dos navegadores $\left(\mathrm{VO}_{2 \text { máx }}: \mathrm{r}=-0,31\right.$; $\mathrm{VO}_{2} 2^{\circ} \mathrm{LV}: \mathrm{r}=-0,31 ; \% 2^{\circ} \mathrm{LV}: \mathrm{r}=-0,43$; Temp_2 $\left.{ }^{\circ} L V: r=-0,52\right)$ com o desempenho em tomada de decisão $(p>0,05)$. Podemos atribuir esses resultados à multidisciplinaridade do esporte, pois além da exigência de adequado nível de aptidão física em diversas modalidades, a irregularidade da prova em relação ao terreno e à duração exige dos atletas uma preparação diferenciada, composta pelo desenvolvimento de diversos tipos de habilidades técnicas, diminuindo o volume do treinamento para uma modalidade específica, ao contrário do que acontece com corredores, ciclistas, triatletas e atletas de $\mathrm{CO}(13)$.

Em outros estudos, a aptidão física foi relacionada, com a resposta psicofisiológica e com a tomada de decisão. Esses estudos demonstram que o condicionamento aeróbio pode interferir favoravelmente no desempenho técnico, nos aspectos cognitivos e emocionais, assim como nas respostas fisiológicas ao estresse físico e mental(4,5-8). Segundo estes autores, os indivíduos com melhor condicionamento sofrem menores níveis de estresse e podem apresentar melhor desempenho cognitivo, técnico e fisiológico. A CA tem como uma de suas principais modalidades a orientação, o que torna esses fatores ainda mais influentes no desempenho da competição.

Duarte et al.(7) compararam a reatividade ao estresse através do nível de condutibilidade da pele durante a realização de um teste de estresse entre dois grupos de militares com níveis de condicionamento aeróbio diferentes, avaliado através dos valores de $\mathrm{VO}_{2 \text { máx }}$. Eles encontraram que os indivíduos bem condicionados apresentaram uma menor reatividade simpática, avaliada pelo NCP, tanto em repouso quanto durante a apresentação do estímulo estressor. De forma semelhante, Ribas e Ribeiro(8) não encontraram diferenças significativas quanto às respostas do TD de pilotos de helicópteros militares divididos pelo nível de aptidão física, no entanto, o custo cardíaco relativo de trabalho, obtido por meio da frequência cardíaca, durante voos de helicóptero, apresentou resposta significativa entre os grupos, sugerindo uma maior reatividade cardíaca ao estresse e um maior desgaste metabólico como resposta antecipatória à fadiga no grupo de menor preparo físico.

Em relação à idade, Boutcher et al.(26) avaliaram a resposta cardiovascular a desafios mentais em indivíduos idosos aerobicamente treinados e destreinados. Apesar dos resultados do teste psicológico não mostrarem diferenças significativas entre os grupos, foram encontrados valores de freqüência cardíaca mais baixa nos indivíduos treinados e as mudanças na pressão diastólica e na pressão sistólica durante os desafios mentais foram, significativamente, maiores nos indivíduos não treinados.

Ando et al.(27) examinaram alterações no tempo de reação do campo visual periférico durante exercício intenso acima do limiar ventilatório e observaram que o tempo de reação aumentou significativamente durante o exercício intenso acima do $2^{\circ} \mathrm{LV}$, quando comparado com os valores avaliados em repouso. Além disso, o aumento do tempo de 
reação do campo visual periférico durante o exercício de alta intensidade foi inversamente proporcional ao $\mathrm{VO}_{2 \text { máx }}$, demonstrando que a alta capacidade aeróbia atenuaria o aumento no tempo de reação do campo visual periférico durante o exercício exaustivo. Estes resultados sugerem que pessoas melhores treinadas poderiam ter mais facilidade $\mathrm{e}$ rapidez também na tomada de decisão, visto que sofreriam menor perda de tempo de reação, o que poderia estar relacionado com um melhor desempenho competitivo. Assim, de acordo com a literatura, a aptidão física poderia favorecer um melhor desempenho na tomada de decisão (performance) do navegador, visto ser esta uma condição bastante influenciada pela experiência do atleta na orientação durante uma competição, assim como, pelo seu preparo psicológico e cognitivo. No entanto, os resultados do presente estudo mostraram fracas correlações entre todos os resultados dos TD1 e TD2 com o nível de aptidão física (TABELA 3). O desempenho dos navegadores nos testes TDs e de suas respectivas equipes durante a competição parece não ter sido influenciado pelas diferenças existentes na aptidão física destes atletas. Estes achados estão em concordância com os estudos de Moyna et al.(28) demonstraram que pequenas alterações neuroendócrinas em resposta ao estresse psicológico são independentes do nível de capacidade aeróbia. Da mesma forma, Spalding et al.(29), verificaram que homens treinados e não treinados não obtiveram diferenças após teste de percepção ao estresse indicando que a alta capacidade aeróbia não reduziu a sensação subjetiva ao estresse.

Adicionalmente, as correlações entre 0 nível de aptidão física e os resultados do TD1 e TD2, e destes com o desempenho na competição de $\mathrm{CA}$ não foram significativas (TABELA 3) $(p>0,05)$. Sob um ponto de vista, isso pode significar a baixa contribuição da tomada de decisão no desempenho final das equipes de CA. No entanto, sabemos na prática que uma navegação eficiente pode representar quilômetros ou horas de prova a menos para os integrantes de uma equipe de CA.

Outra possibilidade que pode explicar as fracas correlações é que o teste de TD utilizado neste estudo pode não ser o mais adequado para esse tipo de população, pois, a avaliação ideal da tomada de decisão dos navegadores durante uma competição de CA seria durante um evento competitivo, todavia, sua realização procurou simular sob uma forma mensurável e reprodutível tal característica. Além disso, tanto o tipo de teste como os critérios utilizados em relação ao tempo de duração ou ao grau de dificuldade escolhido podem não ter sido suficientes para determinar um comportamento semelhante ao vivenciado pelos navegadores durante uma competição. No entanto, este teste foi aplicado, anteriormente, em militares para verificar a relação dos resultados em TD com seu nível de aptidão física $(7,8)$, podendo ser considerado relevante para o tipo de população do presente estudo, devido à semelhança nas atividades realizadas.

Outro fator que pode ter influenciado na baixa relação dos resultados no TD com o desempenho nas provas foi o tipo de teste de esforço físico aplicado entre os TDs, visto que a duração do teste de esforço progressivo máximo em esteira gira em torno de 8 a 12 min. Isso representa um estresse físico diferente para os atletas avaliados em relação à realidade do esporte. Possivelmente, o aumento do tempo do teste físico realizado em intensidade contínua, assemelhando-se mais a pratica do esporte, pudesse interferir nos resultados do TD2. Por exemplo, Reilly e Smith(30), verificaram que o efeito da intensidade do exercício sobre a função mental de atletas de $\mathrm{CO}$, avaliada através de problemas aritméticos, pode ser representado por um "U invertido", onde nas baixas e altas intensidades foram verificados os menores números de acertos. Os maiores números de acertos ocorreram durante exercícios realizados entre 25 e $70 \%$ do $\mathrm{VO}_{2 \text { máx }}$. Por outro lado, apesar dos navegadores em nosso estudo terem realizado um teste progressivo máximo, os TDs foram realizados na situação de repouso após 3 min de recuperação. Esta situação assemelha-se, igualmente, as competições, pois em diversos momentos das provas os atletas necessitam parar ou diminuir o ritmo para conferir a navegação. Além disso, o ritmo de prova nas competições não é 
sempre contínuo, em função dos obstáculos a serem transpostos e tarefas a serem realizadas pelos atletas.

Apesar dos resultados de correlação entre aptidão física e resultados dos TDs terem sido fracos, a associação encontrada entre a idade dos navegadores com o desempenho de suas equipes nas competições de CA foi boa $(r=-$ $0,71, \mathrm{p}<0,05)$. Da mesma forma, Palermo et al(13) encontraram boa correlação entre o desempenho das equipes de $\mathrm{CA}$ em competições e a idade dos navegadores $(\mathrm{r}=$ 0,77). Apesar da idade mais elevada não representar, necessariamente, uma melhor preparação técnica ou experiência, esses resultados reforçam a importância que a navegação e a necessidade de superação de situações adversas têm dentro da competição, podendo ser considerado um fator relevante em relação à predição do desempenho em $\mathrm{CA}$.

Neste sentido, um navegador mais velho poderia apresentar maior tolerância ao estresse, devido à maior experiência em navegação ou então a capacidade em administrar conflitos durante a prova. Dessa forma nos detivemos em avaliar a relação da idade dos navegadores com os resultados do $\mathrm{TD}$, pois suas respostas cognitivas e emocionais durante situações estressantes e adversas podem influenciar o desempenho de toda a equipe. A responsabilidade pela tomada de decisão na orientação é um fator que poderia, também, influenciar no desgaste dos navegadores mais jovens, gerando, possivelmente, maior estresse físico e mental em comparação com os outros competidores.

Em nosso estudo, a idade dos navegadores e as variáveis do TD1, apresentaram baixas correlações (TABELA 3), demonstrando pouca influência da idade sobre o TD executado antes do exercício físico. No entanto, foram encontrados bons valores de correlação entre os valores de idade e as variáveis do TD2, realizado depois do exercício físico (PONTOS_TD2: $r=-0,84$; ACERTOS_TD2: $r=-0,86$; ERROS_TD2: $r$ $=-0,77)(\bar{p}<0,01)$. Esses valores de correlação não se confirmaram em relação a variável PERF_TD2 $(\mathrm{r}=-0,43)$. Neste sentido, os resultados do TD2, realizado após o teste de esforço físico apresentaram relação com a idade dos navegadores. Apesar da maior quantidade de pontos e de acertos no TD2, os indivíduos jovens obtiveram, também, uma maior quantidade de erros (FIGURA 1). A relação entre PONTOS_TD2 e ACERTOS_TD2 com ERROS_TD2, foi forte e significativa $(\mathrm{r}=0,92, \mathrm{p}<\overline{0}, 01)$, para ambas as correlações. Verificamos que o maior número de erros obtido no TD2 em comparação com TD1, pode significar uma maior quantidade de erros durante competição, como efeito do cansaço físico e psicológico dos testes $(\mathrm{p}<0,05)$ (TABELA 2). Isso significa que, durante uma competição, o estresse causado pelas exigências fisiológicas próprias do exercício, pelo ambiente hostil, pela privação de sono e pela função de navegação, seria mais bem administrado pelos navegadores mais velhos, em virtude dos resultados encontrados no TD2.

Adicionalmente, esses resultados indicam que os indivíduos mais jovens, apesar de terem acertado mais depois do teste físico, erraram mais do que os indivíduos mais velhos. Em uma situação real, tal como uma competição, esse fato pode ser prejudicial para o desempenho da equipe, pois um erro de orientação pode representar quilômetros e horas a mais em comparação a outras equipes com navegadores mais experientes, visto que os atletas mais velhos erraram menos perante um estímulo estressor. Portanto, mesmo que a quantidade de acertos e pontos dos mais jovens tenha sido melhor e significativa, esse resultado não está sendo favorável em relação aos erros, pois em competições os erros na tomada de decisão, também, interferem no desempenho competitivo. No programa utilizado para avaliar o TD, a quantidade de erros não participa do cálculo dos resultados. Provavelmente, por esse motivo, a PERF_TD2 não apresentou diferença e nem correlação com a idade.

Em estudos realizados por Pesce et al.(31), foi verificado que atletas de orientação experientes e mais velhos apresentaram maior velocidade de reação e controle da atenção em comparação aos menos experientes. Eccles et al.(32), verificaram que atletas de orientação mais experientes possuíam maior habilidade de consultar a carta topográfica durante deslocamento, enquanto que os menos 
experientes precisavam parar mais vezes e por mais tempo. Essa habilidade foi atribuída a maior capacidade de concentração e atenção, variável que pode ser treinada e que pode aumentar o desempenho em esportes caracterizados pela multiplicidade e variação dinâmica de elementos. Para Guzmán et al.(33), a competência em realizar técnicas de orientação de forma eficiente foi a variável primordial que diferenciou atletas de orientação de elite daqueles que não são elite.

\section{Pontos fortes e limitações do estudo}

Uma importante limitação deste estudo foi o teste físico utilizado para simular uma situação competitiva nas corridas de aventura. O teste progressivo máximo em esteira com intensidades crescentes até atingir a intensidade máxima não se assemelha com a duração, com a variação de intensidade de corrida e nem com o terreno que costuma caracterizar essas competições. Neste sentido, as condições estressantes em que o navegador necessita tomar decisões para orientar sua equipe não são idênticas as que foram submetidos neste estudo. Entretanto, o teste físico escolhido, além de ser de fácil e rápida execução foi suficientemente intenso para gerar uma alteração aguda no resultado do TD2 em comparação ao TD1. Qualquer outro teste que fosse mais próximo das condições de prova despenderia um maior tempo e energia dos seus participantes, além de não submeter os indivíduos sob a verdadeira pressão competitiva.

Dentre as possibilidades de futuros estudos sugere-se a realização de testes físicos sob condições mais próximas das provas de corrida de aventura a fim de verificar seu efeito nos parâmetros de tomada de decisão dos navegadores e no desempenho em tomada de decisão

\section{Conclusão}

Conclui-se que a aptidão física não é parâmetro exclusivo para a determinação do desempenho de atletas de corrida de aventura. Apesar da necessidade de preparação fisiológica adequada a fim de enfrentar o desgaste físico das provas, os aspectos psicológicos e a experiência do navegador parecem influenciar de forma significativa no desempenho final da equipe. Isso pode ser explicado pelo fato da corrida de aventura ser um esporte multidisciplinar, exigindo dos participantes um preparo não só fisiológico, mas também psicológico e cognitivo. Isso nos leva a crer que quanto mais experiente for o navegador, menos erros ele terá durante uma prova de corrida de aventura, proporcionando um menor desgaste físico de todos os componentes da equipe. Isso pode ser bastante relevante, pois quem erra mais, percorre maiores distâncias e perde tempo em relação às outras equipes. Dessa forma, sugere-se que as equipes deem atenção, em seu período de preparação, não somente ao fator fisiológico, mas, também, aos fatores envolvidos com a tomada de decisão e a prática da orientação no planejamento do treinamento para um melhor desempenho nas competições.

\section{Declaração de conflito de interesses}

Não nenhum conflito de interesses no presente estudo.

\section{Referências}

01. Levada-Pires AC, Fonseca CE, Hatanaka E, Alba-Loureiro TD, Angelo A, Velhote FB, et al. The effect of an adventure race on lymphocyte and neutrophil death. Eur J Appl Physiol 2010;109:447-53.

02. Rincón EO, Marchena JM, García JJ, Schmidt A, Schultz T, Malpica I, et al. Phagocytic function in cyclists: correlation with catecholamines and cortisol. J Appl Physiol 2001;91:1067-72.

03. Coyle E. Physical activity as a metabolic stressor. Am J Clin Nutr 2000;72(S):512-20.

04. Achten J, Halson SA, Moseley L, Rayson MP, Casey A, Jeukendrup AE. Higher dietary carbohydrate content during intensified running training results in better maintenance of performance and mood state. J Appl Physiol 2004;96:1331-40.

05. Halson SL, Bridge MW, Meeusen R, Busschaert B, Gleeson M, Jones DA, et al. Time course of performance changes and fatigue markers during intensified training in trained cyclists. J Appl Physiol 2002;93:947-56.

06. Wasmund LW, Westerholm EC, Watenpaugh DE, Wasmund SL, Smith ML. Interactive effects of mental and physical stress on cardiovascular control. J Appl Physiol 2002;92:1828-34. 
07. Duarte AF, Pitaluga MV, Moraes JM, Ribeiro LCS. Condicionamento físico aeróbio e reações psicofisiológicas a um teste de estresse. Rev. Educ. Física 2003;127:4-9.

08. Ribas PR, Ribeiro LCS. Aptidão física e o controle do comportamento psicofisiológico de pilotos de helicópteros do Exército Brasileiro pelo biofeedback. Rev. Educ. Física 2003;127:41-7.

09. Anglem N, Lucas SJE, Rose EA, Cotter JD. Mood, illness and injury responses and recovery with adventure racing. Wild Environ Med 2008;19:30-8.

10. Lucas SJ, Anson JG, Palmer CD, Hellemans IJ, Cotter JD. The impact of 100 hours of exercise and sleep deprivation on cognitive function and physical capacities. J Sports Sci 2009;27:719-28.

11. Mann D, Schaad K. The complete guide to adventure racing. Nova Iorque: Hatherleigh Press; 2001.

12. Dias AC, Dantas EHM. A tomada de decisão na orientação. Rev. Educ. Física 2004;128:12-7.

13. Palermo MA; Coelho FD; Gorski T; Coertjens M; Kruel LFM. Relação entre o condicionamento físico e a idade no desempenho de equipes de corrida de aventura. Revista Kinesis, v. 31, p. 5574, 2013.

14. Tanaka K, Matsuura Y, Matsuzaka A, Hirakoba K, Kumagal S, Sun SO, et al. A longitudinal assessment of anaerobic threshold and distance-running performance. Med Sci Sports Exerc 1984;16:278-82.

15. Péronnet F, Thibault G, Rhodes EC, McKenzie DC. Correlation between ventilatory threshold and endurance capability in marathon runners. Med Sci Sports Exerc 1987;19:610-15.

16. Maffulli N, Capasso G, Lancia A. Anaerobic threshold and performance in middle and long distance running. J Sport Med Phys Fit 1991;31:332-38.

17. Sleivert GG, Wenger HA. Physiological predictors of short-course triathlon performance. Med Sci Sports Exerc 1993;25:871-6.

18. Taylor HL, Buskirk E, Henschel A. Maximal oxygen intake as an objective measure of cardiorespiratory performance. J Appl Physiol 1955;8:73-80.

19. Amann M, Subudhi A, Foster C. Influence of testing protocol on ventilatory thresholds and cycling performance. Med Sci Sports Exerc 2004;36:613-22.
20. Gaskill SE, Ruby BC, Walker AJ, Sanchez OA, Serfass RC, Leon AS. Validity and reliability of combining three methods to determine ventilatory threshold. Med Sci Sports Exerc 2001;33:1841-8.

21. Creagh U, Reilly T. Physiological and biomechanical aspects of orienteering. Sports Med 1997;24:409-16.

22. Bassett DR, Howley ET. Limiting factors for maximum oxygen uptake and determinants of endurance performance. Med Sci Sports Exerc 2000;32:70-84.

23. Noakes TD. Physiological models to understand exercise fatigue and the adaptations that predict or enhance athletic performance. Scand J Med Sci Sports 2000;10:123-45.

24. Butts NK, Henry BA, Mclean D. Correlations between $\mathrm{VO}_{2 \text { máx }}$ and performance times of recreational triathletes. J Sport Med Phys Fit 1991;31:399-44.

25. Bulbulian R, Wilcox AR, Darabos BL. Anaerobic contribution to distance running performance of trained cross-country athletes. Med Sci Sports Exerc 1986;18:107-13

26. Boutcher SH, Nurhayati Y, Mclaren PF. Cardiovascular response of trained and untrained old men to mental challenge. Med Sci Sports Exerc 2001;33:659-64.

27. Ando S, Kimura T, Hamada T, Kokubu M, Moritani $\mathrm{T}$, Oda $\mathrm{S}$. Increase in reaction time for the peripheral visual field during exercise above the ventilatory threshold. Eur J Appl Physiol 2005;94:461-67.

28. Moyna NM, Bodnar JD, Goldberg HR, Shurin MS, Robertson RJ, Rabin BS. Relation between aerobic fitness level and stress induced alterations in neuroendocrine and immune function. Int $\mathrm{J}$ Sports Med 1999;20:136-41.

29. Spalding TW, Jeffers LS, Porges SW, Hatfield BD. Vagal and cardiac reactivity to psychological stressors in trained and untrained men. Med Sci Sports Exerc 2000;32:581-91.

30. Reilly T, Smith D. Effect of work intensity on performance in a psychomotor task during exercise. Ergonomics 1986;29:601-06.

31. Pesce C, Cereatti L, Casella R, Baldari C, Capranica L. Preservation of visual attention in older expert orienteers at rest and under physical effort. J Sport Exerc Psychol 2007;29:78-99. 
32. Eccles DW, Walsh SE, Ingledew DK. Visual attention in orienteers at different levels of experience. J Sports Sci 2006;4:77-7.

33. Guzmán JF, Pablos AM, Pablos C. Perceptualcognitive skills and performance in orienteering. Percept Mot Skills 2008;107:159-64. 